Document downloaded from:

http://hdl.handle.net/10251/125668

This paper must be cited as:

Sanchis-Cano, A.; Guijarro, L.; Pla, V.; Vidal Catalá, JR. (2019). Duopoly Provision of Services Based on Wireless Sensor-supplied Data: a Differential Game Model. Mobile Networks and Applications (Online). 1-11. https://doi.org/10.1007/s11036-018-1128-6

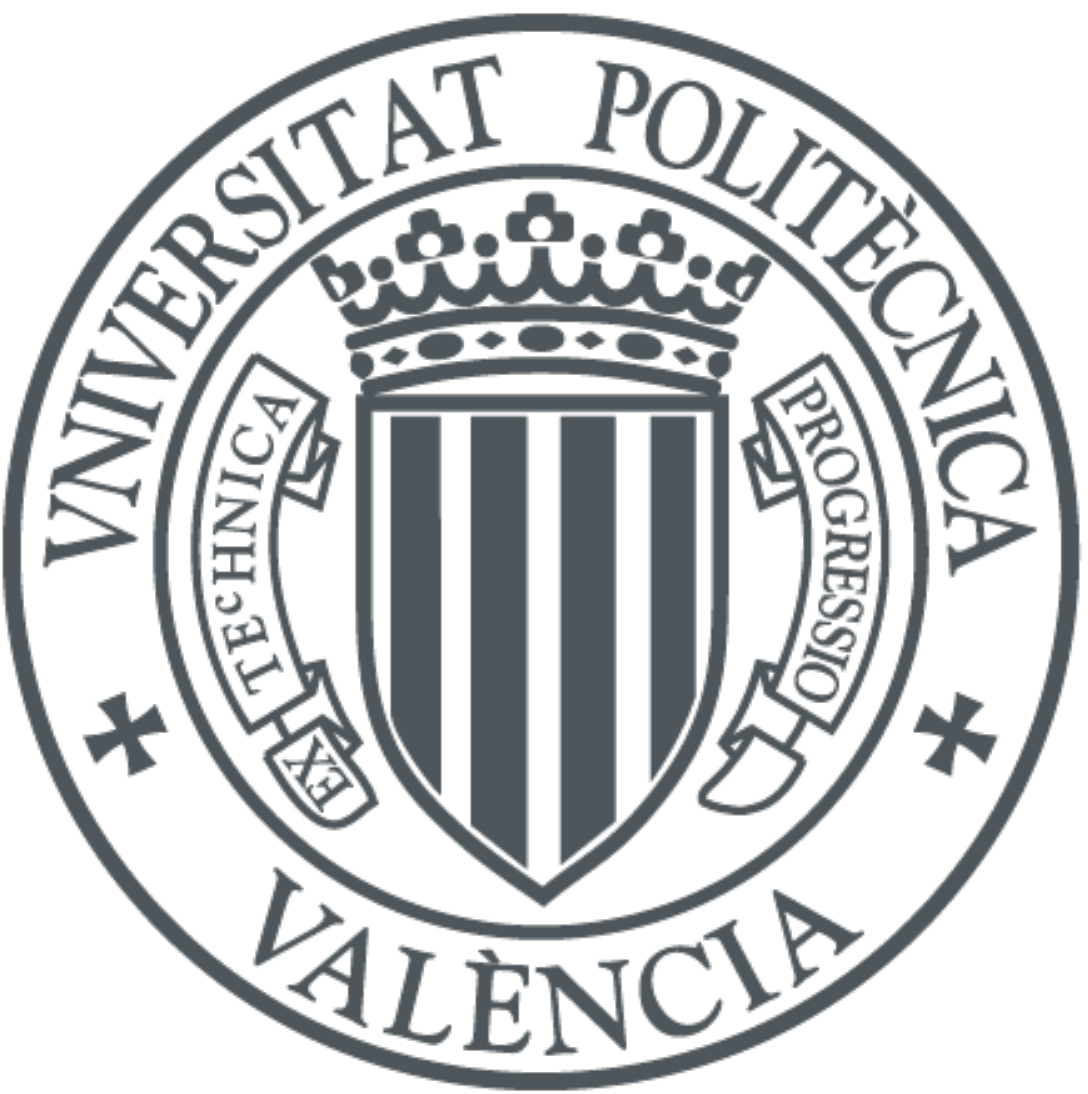

The final publication is available at

http://doi.org/10.1007/s11036-018-1128-6

Copyright Springer-Verlag

Additional Information 


\title{
Duopoly provision of services based on wireless sensor-supplied data: a differential game model
}

\author{
Angel Sanchis-Cano - Luis Guijarro - Vicent Pla · Jose R. Vidal
}

Received: date / Accepted: date

\begin{abstract}
The provision of services based on wireless sensor data is analyzed dynamically. In the proposed scenario, two competing service providers deploy their own wireless sensor networks in order to collect the data and provide services to final users. The service providers compete in prices dynamically in order to maximize their profits, while the behavior of the users is modeled using a discrete choice model. The model is analyzed using game theory. The changes in the population of users are analyzed through an evolutionary game and the Logit dynamic, while the dynamic competition in prices is studied using a differential game. We conclude that the dynamic pricing competition is economically feasible for the service providers and, in addition, the dynamic solution converges to the static solution of the scenario.
\end{abstract}

Keywords wireless sensor networks - network economics · dynamic pricing - differential game $\cdot$ internet of things · game theory

\section{Introduction}

The world is moving from a standalone devices scenario to a all-connected scenario also known as Internet of Things (IoT). The basic idea is to have connected almost all objects around us, being able to communicate with each other and connected to the Internet. The relevance of the IoT is growing due to the increasing number of connected devices [1]. In the Ericsson Mobility Report it is estimated that the number of devices connected by 2020 will be 26 billion,

Angel Sanchis-Cano $\cdot$ Luis Guijarro · Vicent Pla $\cdot$ Jose R. Vidal ITACA. Universitat Politècnica de València. Camino de Vera s/n. 46022 Valencia, Spain

Angel Sanchis-Cano E-mail: ansanca8@itaca.upv.es

This work was supported by the Spanish Ministry of Economy and Competitiveness through project TIN2013-47272-C2-1-R and co-supported by the European Social Fund BES-2014-068998. and only 6.6 billion will be human type devices [2]. Several new problems must be solved in order to be able to serve this growing number of IoT connected devices [3], from the development of new technologies and protocols [4], to the y economic analysis of the emerging scenarios [5].

In this paper we are centered in the economic analysis. This analysis uses several tools, and the pricing is one of the most extended. It has been used successfully in networks to avoid or mitigate congestion scenarios [6] as well as a tool to improve the energy usage of the devices [7]. For instance, the work in [8] proposes a pricing mechanism to avoid the congestion due to massive Machine Type Communication (MTC) accesses, increasing the price of the service when the network usage increases. Reference [9] proposes a model where a centralized system is implemented to control the channel sharing in cognitive radio networks based on a credits system and how this mechanism allows to achieve different sharing objectives.

Network pricing is a useful tool for congestion control, but also is a tool needed in the study of the economic viability of a scenario. It helps us to obtain the equilibrium prices where supply and demand are balanced and the operator profits are maximized [10]. To study the economic viability of a network scenario the users utility and the operators profits should be known, and consequently a pricing scheme is needed. In this research line there are several examples, from simple scenarios, where a monopolistic operator offers several services with different pricing policies, based on the quality of each service [11], to more complex scenarios, where two or more operators compete for serving users while they try to maximize their own profits. In these cases the study of competition is needed. Historically, the concepts of the economic analysis of transport systems field [12], based on game theory approaches, have been applied to the competition in the networks field [13]. Many works study the competition 
between operators with several considerations. Some studies analyze the competition in models with homogeneous traffic profiles [14], and also that analyze the competition between operators offering heterogeneous services like [15].

Typically, the economic viability was studied in network connectivity provision scenarios, however, due to the growing of the IoT and the WSN, the study of provision of different services over the network is becoming more relevant. The economic analysis of the services provision allows us to study how the IoT data could be monetized, and therefore study the economic viability from the point of view of actors other than the operators, such as the IoT devices owner. The works in this line are mainly focused in static scenarios, where the conditions of the scenario remain fixed and the solution obtained is the static equilibrium [16]. However, there are studies that go one step further, analyzing evolving scenarios, where the analyzed conditions change over time, and therefore, the optimal strategies [17-19]. Nevertheless, these works do not study the pricing problem dynamically.

Our work extends the analysis performed by the authors in [20], where the analysis was based on two coupled one-shot games. In the second game two Internet of Things Service Providers (IoT-SPs) compete to provide sensor-data-based services to their clients, the final users. In the first game the sensors compete to transmit the data gathered to their IoT-SP through the access network of a Network Operator (OP) and Internet. Here the analysis goes further and models and analyzes a dynamic competition between the service providers.

Our main contribution is to show that the dynamic competition in prices is economically viable for all the actors in the market. In addition, we also show how the initial distribution of users in the market alters the profit of the IoT-SPs and how the number of sensors deployed by each IoT-SP modifies the profits obtained by the providers.

The model described in this paper is valid for time sensitive applications. One possible application is a weather alert system, where the weather sensors are our sinks, that transmit the weather data in different places through an OP network. The service provider gathers that data of their sensors and offers a bad weather alert service to the people or companies that subscribe to it, which are the users of our model. This scheme is also valid for other kind of applications, such as traffic jams monitoring.

The structure of this paper is the following: in Section 2, it is described the model analyzed with the actors involved, their behavior and the monetary flow. In Section 3, the model is described in terms of game theory, where two games are proposed to solve the sensor data acquisition and the users' service provision. Section 4 shows and explains the results. Finally, Section 5 presents the conclusions.

\section{General Model}

Fig. 1 depicts the scenario under analysis in this paper. There are two competing IoT-SPs providing services based on wireless sensor-supplied data to final users using their WSNs. The sensors are grouped into clusters using Machine to Machine communications (M2M) and are owned by one IoT-SP. All the data obtained in a cluster is transmitted to their IoT-SP server (IoT-SP $i$ srv) by the sink node, using the connectivity service of the OP and Internet. The data stored in the IoT-SP servers is is used to provide the services. In order to improve the readability of the paper, we are going to explain briefly all the elements of the model. The actors identified in our model are:

- Sinks.

- Network Operator.

- Final users.

- Internet of Things-Service Providers.

\subsection{Sinks}

The sinks subscribe to the connectivity service of the OP in order to transmit the gathered data to their IoT-SP. The connectivity service received by the sinks is modeled through the quality function $Q(t)=c \frac{\tau}{T(t)}$ [11], where $\tau$ is the mean sensing-data-unit (s.d.u) transmission time and $\mathrm{T}$ is the mean s.d.u service time, obtained for an OP service modeled as an M/M/1 system. This quality function decreases when the delay of the transmitted data increases, therefore is valid for time sensitive applications such as real time traffic or weather monitoring. The sinks' behavior is modeled using the quality perceived for the connectivity service minus the price charged by the OP to the users that subscribe their connectivity service, trough the well known compensated utility function [21]:

$U_{s}(t) \equiv Q(t)-p(t)=c\left(1-x_{1}(t) \tau N r\right)-p(t)$,

where $c$ is a conversion factor, $x_{1}$ is the fraction of sinks subscribed to the OP, $r$ is the sink data generation rate, $N=N_{1}+N_{2}$ is the total number of sinks and where $N_{j}$ is the number of sinks of the $\operatorname{IoT}_{-S_{j}}$ and $p(t)$ is the price in monetary units (m.u) that each $\operatorname{IoT}_{-S P}(j=1,2)$ pays to the OP when its sinks transmit one s.d.u. Note that the IoT-SP is who pays to the OP, however, the subscription decision is made individually by each sink. Assuming that the sinks that do not subscribe to the service perceive zero utility, equation (1) models that the users will subscribe to the service only if the quality of the service perceived is not lower than the price charged by the OP.

The sinks' distribution is represented by $X_{s}(t)=$ $\left(x_{0}(t), x_{1}(t)\right)$, where $x_{0}$ and $x_{1}$, are the fraction of sinks 


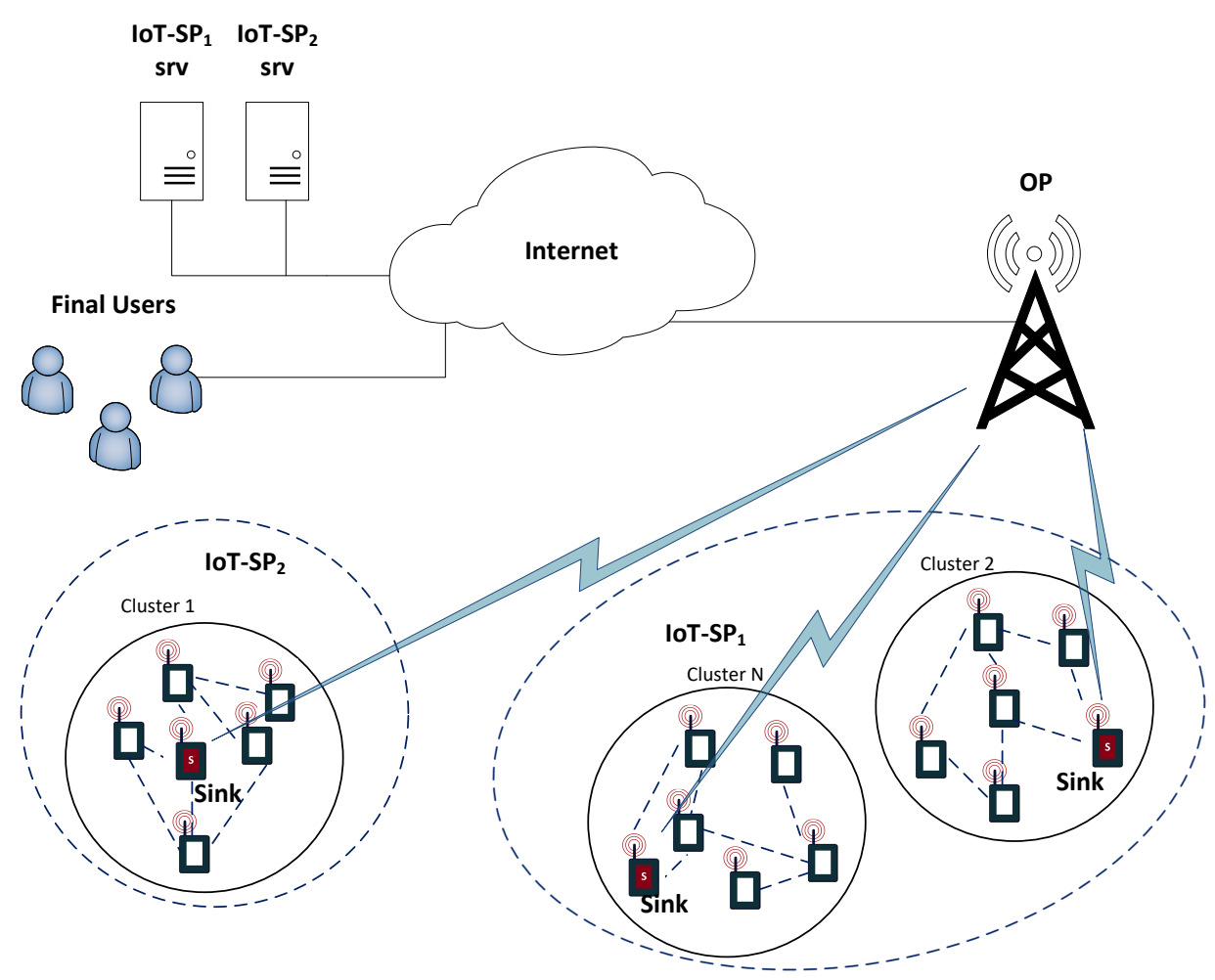

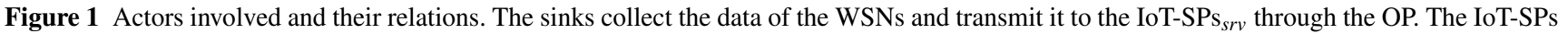
process the data in the servers in order to offer the sensing data-based services to the final users.

subscribed and not subscribed to the OP connectivity service respectively and $\sum_{i=0,1} x_{i}(t)=1$.

\subsection{Network Operator}

The OP provides a connectivity service to the sinks. Each IoT-SP pays a price $p(t)$ to the OP per s.d.u. transmitted by its sinks. Therefore, the OP instantaneous profit at time $t$ can be obtained as price at time $t, p(t)$ multiplied by the amount of data transmitted by all the sinks subscribed to the service in the same time instant:

$\Pi_{O P_{i n s}}(t)=p(t) x_{1}(t) r N$.

\subsection{Users}

The users subscribe to the sensing-data-based service of the IoT-SPs. The preferences a user $j$ are modeled using the Logit discrete choice model and a utility function based on [19]:

$U_{u_{j}}(t)=\varphi \log \left(R_{j}(t)-f_{j}(t)\right)+\kappa_{u_{j}}$,

where $\kappa_{u_{j}}$ is random variable that follows a Gumbel distribution of mean 0 that models the unobserved individual user preferences, such as the fidelity of the users with a specific company, $R_{j}(t)=x_{1}(t) r N_{j}$ is the data rate generated by the sinks of the IoT-SP ${ }_{j}$ and transmitted to them through the OP and Internet, $f_{j}(t)$ is the price in m.u per second that the IoT-SP ${ }_{j}$ charges to the users for its service and $\varphi>0$ is a sensitivity parameter that models the relative importance of the difference $R_{j}(t)-f_{j}(t)$. In this case, the users' utility is better if the data rate $R_{j}$ increases and worst when the price charged by the IoT-SP $j, f_{j}(t)$, increases. In addition, it has been proved through the Weber-Fechner law, that the human perception of the physical stimuli use to be logarithm [22], which justifies the usage of the logarithm in the utility function.

Assuming that the users are rational players, they will choose the strategy (IoT-SP ${ }_{j}$ ) that maximizes their utility $U_{u_{i}}(t) \geq U_{u_{j}}(t) \forall j \neq i$. The total number of users is $M$, while the users' distribution is described by $X_{u}(t)=\left(y_{1}(t), y_{2}(t)\right)$, where $y_{j}(t)$ is the fraction of users that subscribes to IoT-SP $_{j}$. Note that $y_{1}(t)+y_{2}(t)=1$, and that every user subscribes to one IoT-SP.

\subsection{IoT-SPs}

The IoT-SPs collect the data of their sensors in order to provide a data-based service to their users. The IoT-SP ${ }_{j}$ pays a price $p$ to the OP every time its sensors transmit one s.d.u. On the other hand, the users subscribed to the $\operatorname{IoT}_{-} \mathrm{SP}_{j}$ pay a 
price $f_{j}(t)$ every time unit. The IoT-SP ${ }_{j}$ instantaneous profit is:

$\Pi_{I o T-S P_{i n s}}(t)=y_{j}(t) M f_{j}(t)-R_{j}(t) p(t)$.

In the first part of the equation $\left(y_{j}(t) M f_{j}(t)\right)$ we observe the revenues obtained from the users subscribing to the service, while the second part $\left(R_{j}(t) p(t)\right)$ are the costs of keeping the sinks transmitting data through the OP.

\section{Game Analysis}

The scenario described in the previous section is modeled and analyzed using game theory with two different games connected through the value of $R_{j}$. The structure of the two games is similar: firstly, there is a pricing stage, where the operator and providers choose their pricing decisions and secondly a subscription stage, where the sinks and users decide to subscribe or not based on the utility perceived. The structure of the games is shown in Figure 2.

In this scenario we can assume that the changes in Game I, for instance in the price charged to the sinks or the deployment of WSNs, are much less frequent than the changes in Game II, such as the variation in the price charged to the users and the number of users in the market. In this case, we can consider that most of the time, the outcome of Game I is constant while Game II is being played. In addition, the outcome of Game I is a stationary point that matches the Nash equilibrium obtained in [20], where the game is analyzed statically. On the other hand, the changes in Game II, and particularly the prices, are relatively frequent, and therefore, the equilibrium solution is a function of time, which is obtained using differential games [23].

\subsection{Game I: Sinks and OP}

In this game, the sinks make their subscription decision to the OP service to transmit the collected sensing data to their IoT-SP based on the utility perceived, while the OP chooses the price $p$ that maximizes its profit. Given that we can consider Game I static from the point of view of Game II the solution is those obtained in [20]:

$$
\begin{aligned}
& \Pi_{O P}^{*}= \begin{cases}c(1-\tau N r) N r & \text { if } \tau<\frac{1}{2 N r} \\
\frac{c}{4 \tau} & \text { if } \frac{1}{2 N r} \leq \tau\end{cases} \\
& p^{*}=\left\{\begin{array}{ll}
c(1-\tau N r) & \text { if } \tau<\frac{1}{2 N r} \\
\frac{c}{2} & \text { if } \frac{1}{2 N r} \leq \tau
\end{array} .\right. \\
& x_{1}^{*}= \begin{cases}1 & \text { if } \tau<\frac{1}{2 N r} \\
\frac{1}{2 \tau N r} & \text { if } \frac{1}{2 N r} \leq \tau\end{cases}
\end{aligned}
$$

\subsection{Game II: Users and IoT-SPs}

In this game we analyze the competition in prices between the two IoT-SPs in order to maximize their profit and the subscription of the users to the IoT-SPs service. This game is analyzed both statically and dynamically assuming the static solution for Game I obtained above.

In the static analysis we obtain the Stackelberg equilibrium, where the IoT-SPs are the leaders and the users are the followers. The pricing strategies of the competing IoT-SPs are obtained using the concept of Nash equilibrium, while the users' subscription decision is obtained through the Logit probability of choice.

The objective of the dynamic analysis is to analyze more realistic scenarios, where the optimal decisions may vary over time due to changing conditions. In order to obtain more general results and restrictions we use continuous time unless otherwise specified. The dynamic analysis is conducted using evolutionary game theory for the Users Subscription Game and differential games for the IoT-SPs pricing stage.

In order to be able to compare the results we first analyze the static solution of the game, and once it is obtained we proceed with the dynamic solution.

\subsubsection{Static Analysis}

In this stage we analyze the equilibrium reached when all the system parameters remain static and the actors can make their decision only once. Under such circumstances we can consider the equations defined in previous sections without time dependency.

\section{Users Subscription Stage}

In this stage the users decide which IoT-SP to subscribe. The behavior of the users is modeled by the utility function described in (3), which is a Logit discrete choice model. In the logit model, we can obtain the probability of a user choosing the $\mathrm{IoT}_{-\mathrm{SP}}$ as :

$$
\omega_{j}=\frac{e^{\log \left(R_{j}-f_{j}\right)}}{\sum_{k=1}^{2} e^{\log \left(R_{k}-f_{k}\right)}} .
$$

If we consider a number of users large enough, the portion of users subscribing with the IoT-SP $i$ equals the probability of a user choosing that IoT-SP. Therefore, we obtain:

$$
\begin{aligned}
& y_{1}=\frac{R_{1}-f_{1}}{\left(R_{1}-f_{1}\right)+\left(R_{2}-f_{2}\right)}, \\
& y_{2}=\frac{R_{2}-f_{2}}{\left(R_{1}-f_{1}\right)+\left(R_{2}-f_{2}\right)} .
\end{aligned}
$$




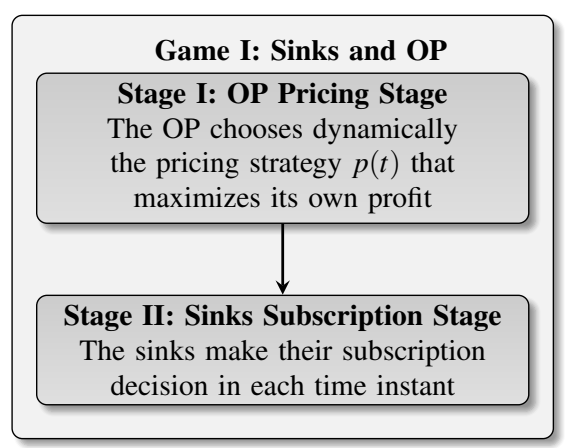

Game II: Users and IoT-SPs

\section{Stage I: IoT-SPs Pricing Stage}

Both IoT-SPs compete dynamically

with their pricing strategies $f_{j}(t)$

in order to maximize their profits

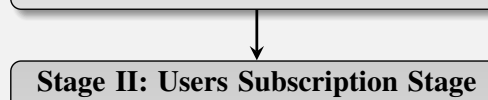

Stage II: Users Subscription Stage

Final users decide to subscribe

with a IoT-SP in each time

instant given the utility perceived

Figure 2 Structure of the games and description of the stages in each game.

\section{IoT-SPs Pricing Stage}

Given the profit of the IoT-SPs described in (4) and the solution of the Users Subscription Stage (9), we can rewrite the profit of the IoT-SPs as:

$\Pi_{I o T-S P_{i}}\left(f_{1}, f_{2}\right)=\frac{f_{1} M\left(f_{1}-R_{1}\right)}{f_{1}+f_{2}-R_{1}-R_{2}}-p R_{1}, \quad i=1,2$.

In this case, the equilibrium reached is the Nash equilibrium, which can be obtained as the crossing point of the best response functions. In our scenario we can define the best response as the best strategy of IoT-SP $_{i}$ given a strategy of $\operatorname{IoT} \mathrm{SP}_{j}(i \neq j)$ :

$$
\begin{gathered}
B R_{i}\left(f_{j}\right)=f_{i}^{*}\left(f_{j}\right)=\underset{f_{i}>0}{\operatorname{argmax}} \Pi_{I o T-S P_{i}}\left(f_{i}, f_{j}\right), \\
i, j \in\{1,2\}, i \neq j,
\end{gathered}
$$

and the Nash equilibrium is obtained solving the following equations system:

$f_{1}^{*}=B R_{1}\left(f_{2}^{*}\right)$

$f_{2}^{*}=B R_{2}\left(f_{1}^{*}\right)$.

Fig. 3 shows the representation of the best responses and the Nash equilibrium. Note than in this case, there is only one equilibrium, given that the analytical solution of the best responses represented in the figure is only valid for $0<f_{2}<$ 100 and $0<f_{1}<200$.

\subsubsection{Dynamic Analysis}

The objective of the dynamic analysis is to analyze a more realistic scenario, where the system parameters such as the number of sinks or the number of users may vary over time. Therefore, the static equilibrium obtained in the previous stage is not a valid solution, given that the optimal strategy for all the actors may evolve with time.

In these scenarios the pricing strategies of the IoT-SPs are functions of time and may evolve, in addition, the classical tools employed to find static Nash equilibria are not a valid. Under such circumstances, where the parameters

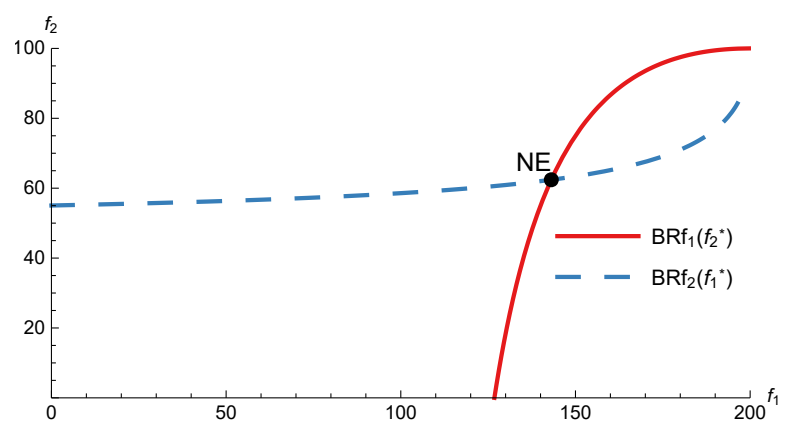

Figure 3 Representation of best responses and Nash Equilibrium (NE) in a particular case.

of the system, the strategies of the other IoT-SP and the distribution of users change over time we need to use a new mathematical framework known as differential games $[17,23]$.

Related with the users, in the static analysis we have considered that all the users make their decisions once for all, which is not realistic. In order to analyze a more realistic behavior the users are modeled using an evolutionary game, where the population of users change their decisions progressively until they reach the optimal strategy following an evolutionary dynamic. In addition, this dynamic allows the users to change their decisions when the prices announced by the IoT-SPs change, which is a requirement for the differential game.

\section{Users Subscription Stage}

In order to maximize dynamically the utility of the users defined in (3), we define the following game:

- Strategies: $S=\left\{S_{1}, S_{2}\right\}$, where $S_{j}$ means to subscribe to the IoT-SP ${ }_{j}$ service.

- Social State: $X_{u}(t)=\left\{y_{1}(t), y_{2}(t)\right\}, y_{1}+y_{2}=1$. Users' distribution between IoT-SPs.

- Payoffs: $F_{s}(t)=\left\{U_{u_{1}}(t), U_{u_{2}}(t)\right\}$. Note that the utility is a function of time, given that the social state and the prices also vary with time.

The previous game is modeled using a evolutionary dynamic, that represents the evolution over time of the 


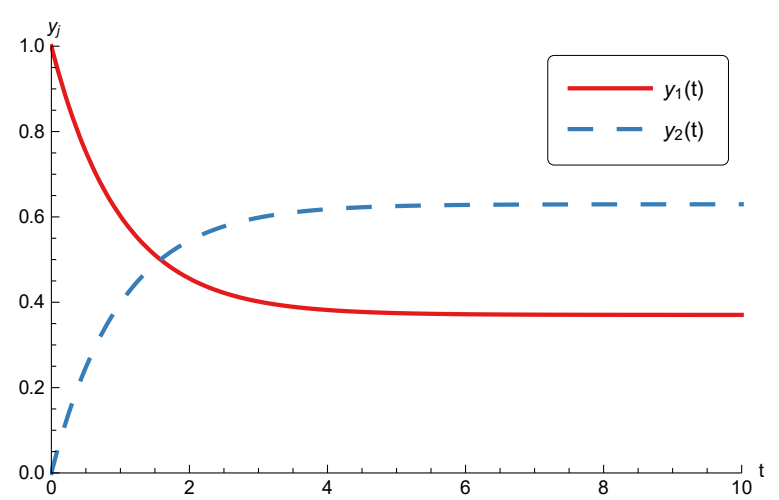

Figure 4 Evolution of the Logit dynamic when $R_{1}=15, R_{2}=20$, $f_{1}=5, f_{2}=3, \delta=1, y_{1}(0)=1$ and $y_{2}(0)=0$

population of users. The usage of evolutionary dynamics allows us to model realistic users, with limited information and inertia in their global behavior. The dynamic of the users is:

$\dot{y}_{i}=\delta\left(\omega_{i}-y_{i}\right)$,

where $\delta$ is the learning rate, $\omega_{i}$ is the logit probability of choosing the strategy $i$ defined in (8) and $\dot{y}_{i}$ represents the instantaneous variation of the population of users subscribed to the IoT-SP ${ }_{i}$. This mean dynamic is known as the Logit dynamic $[19,24]$. Note that in this dynamic, the value of $y_{j}$ converges to $\omega_{i}$, however, $y_{i}=\omega_{i}$ is only reached in the stationary states. Substituting the values of our model in (13), we obtain the instantaneous variation of the population of users subscribed to each IoT-SP:

$\dot{y}_{1}=\delta\left(\frac{R_{1}-f_{1}}{\left(R_{1}-f_{1}\right)+\left(R_{2}-f_{2}\right)}-y_{1}\right)$

$\dot{y}_{2}=\delta\left(\frac{R_{2}-f_{2}}{\left(R_{1}-f_{1}\right)+\left(R_{2}-f_{2}\right)}-y_{2}\right)$.

Figure 4 shows the evolution of the population of users as a function of time, when their behavior is modeled by (14).

The Logit dynamic, unlike the replicator dynamic, has the property that the pure strategies are not necessarily steady states, as shown in Figure 5, where we observe the evolution of the Logit dynamic in a particular case with four different initial states, where the Globally Evolutionary Stable Strategy (GESS) is the mixed strategy equilibrium.

\section{IoT-SPs Pricing Stage}

In this stage, each IoT-SP tries to maximize its profits within a time horizon $t \in[0, T]$, and not only maximizing its instantaneous profits in every time instant. In addition, it is common to introduce a discount rate $\eta$, which modifies value of future payoffs in the present moment. The aggregated profit functions of the IoT-SPs are

$\Pi_{I o T-S P_{j}}=\int_{0}^{T} e^{-\eta t} \Pi_{I o T-S P_{i n s_{j}}} d t . \quad j=1,2$.

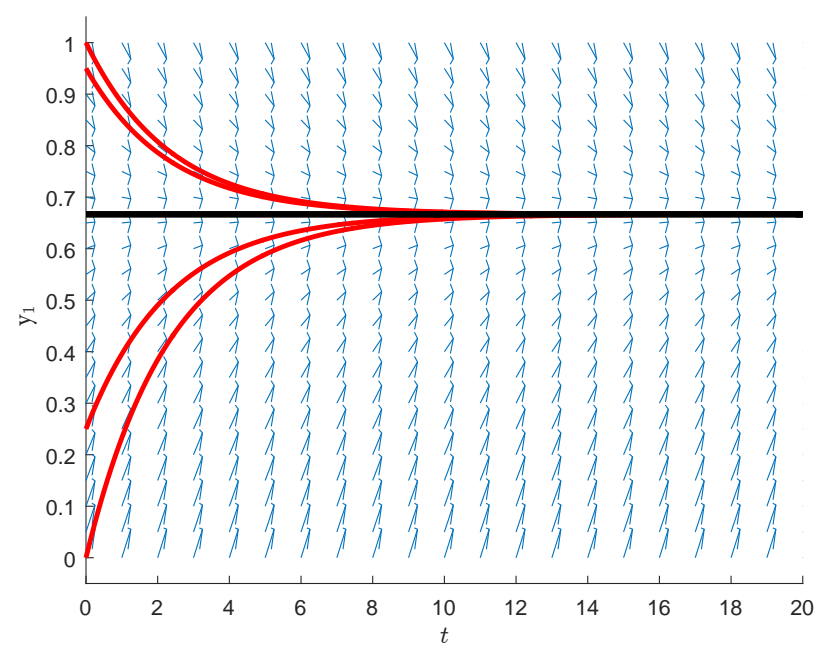

Figure 5 Convergence of the Logit dynamic in the case where a mixed equilibrium is the GESS.

The objective of the IoT-SPs is to maximize their aggregated profits, where the price charged to the users $f_{j}$ is the maximization variable. In this case, both IoT-SPs have conflicting interests and therefore, this cannot be solved as two independent maximization problems. This strategic interaction in static scenarios is solved using the Nash equilibrium however, in dynamic problems, one of the equilibrium concepts employed is the Open-Loop Nash Equilibrium (OLNE). In order to solve the OLNE for dynamic problems we introduce the concepts of optimal control problems and differential games. An optimal control problem allows us to solve problems with restrictions such as (15), where the optimal strategy is not a static value, but a function of time, also known as optimal path. When there are several actors with conflicting interests solving an optimal control problem each one we have a differential game. In order to solve the optimal path within a time horizon $t \in[0, T]$ of both competing IoT-SPs, each one solves an optimal control problem that maximizes its profit given the strategies of the other IoT-SP and the behavior of the users. The formulation of the optimal control problem for each IoT-SP is:

$$
\begin{aligned}
\max _{f_{j}} \Pi_{I o T-S P_{j}}= & \int_{0}^{T} e^{-\eta t} \Pi_{I o T-S P_{i n s_{j}}} d t, \\
\text { s.t } \quad & \dot{y}_{1}=\delta\left(\omega_{1}-y_{1}\right), \\
& \dot{y}_{2}=\delta\left(\omega_{2}-y_{2}\right), \\
& X_{u}(0)=\left\{y_{1,0}, y_{2,0}\right\}, \\
& f_{i},(i=1,2) \in \mathbb{R}_{>0},
\end{aligned}
$$

where $\Pi_{I o T-S P_{i n s_{j}}}$ is the instantaneous profit of the IoT-SP shown in (4), $\dot{y}_{j}$ is the instantaneous variation of the population of users shown in (14) given the strategies of both IoT-SPs and $X_{u}(0)$ is the initial distribution of the population of users. 
In order to solve the maximization problem with restrictions defined in (3.2.2) we used the Pontryagin's Maximum Principle (PMP), which defines the necessary conditions that the candidate optimal strategies must meet. The solution obtained in this paper is the open-loop solution. The IoT-SPs' Hamiltonian function is:

$H_{1}\left(f_{1}, f_{2}\right)=\Pi_{I o T-S P_{i n s_{1}}}+\sigma_{11} \dot{y}_{1}+\sigma_{12} \dot{y}_{2}$

$H_{2}\left(f_{1}, f_{2}\right)=\Pi_{I o T-S P_{i n s_{2}}}+\sigma_{22} \dot{y}_{2}+\sigma_{21} \dot{y}_{1}$.

where $\sigma_{j i}$ are the adjoint variables of the OP, that model the changes in the population state as a consequence of the variation in the prices of all the IoT-SPs. The PMP necessary conditions for the IoT-SP ${ }_{j}$ are:

$f_{j}^{*}(t)=\max _{\left.f_{j} \in\right] 0, \mathbb{R}^{+}[} H_{j}$,

$\dot{y}_{j}=\delta\left(\omega_{j}-y_{j}\right)$,

$\dot{\sigma}_{j i}=\sigma_{j i} \eta-\frac{\partial H_{j}}{\partial y_{i}}$

$\sigma_{j i}(T)=0$.

where $i, j=1,2$, (16) is the maximality condition, (17) is the Logit dynamic, which models the evolution in the population of users, (18) are the adjoint equations and (19) are the transversality conditions. Solving (16) we obtain the candidate strategies $f_{1}^{*}, f_{2}^{*}$ to optimal paths in terms of the state $y_{1}, y_{2}$ and the adjoint variables $\sigma_{11}, \sigma_{12}, \sigma_{22}, \sigma_{21}$. Replacing the optimal candidate strategies $f_{1}^{*}, f_{2}^{*}$ in the remaining PMP conditions and with the initial state conditions we have the following system of PDE:

$$
\begin{cases}\dot{y}_{1} & =\delta\left(\omega_{1}^{*}-y_{1}\right), \\ \dot{y}_{2} & =\delta\left(\omega_{2}^{*}-y_{2}\right), \\ \dot{\sigma}_{11} & =\sigma_{11} \eta-\frac{\partial H_{1}^{*}}{\partial y_{1}} \\ \dot{\sigma}_{12} & =\sigma_{12} \eta-\frac{\partial H_{1}^{*}}{\partial y_{2}} \\ \dot{\sigma}_{22} & =\sigma_{22} \eta-\frac{\partial H_{2}^{*}}{\partial y_{2}} \\ \dot{\sigma}_{21} & =\sigma_{21} \eta-\frac{\partial H_{2}^{*}}{\partial y_{1}} \\ y_{1}(0) & =y_{1,0} \\ y_{2}(0) & =y_{2,0} \\ \sigma_{11}(T) & =0 \\ \sigma_{12}(T) & =0 \\ \sigma_{22}(T) & =0 \\ \sigma_{21}(T) & =0\end{cases}
$$

where $\omega_{i}^{*}$ and $H_{i}^{*}$ are the probability of choice the strategy $i$ and the Hamiltonian function of the operator $i$ when prices $f_{1}, f_{2}$ are replaced by the candidates to optimal paths $f_{1}^{*}, f_{2}^{*}$.

The system (20) is a Two Point Boundary Value Problem (TPBVP) that models the solution to the differential game, and cannot be solved using traditional methods for PDEs. We have solved the TPBVP numerically using the shooting
Table 1 Game I parameters

\begin{tabular}{lcc}
\hline Game I parameter & Scenario 1 & Scenario 2 \\
\hline Conversion factor $\left(c\left[\frac{m . u}{s . d . u}\right]\right)$ & 1 & 1 \\
Sink data generation ratio $\left(r\left[\frac{s . d . u}{s}\right]\right)$ & 1 & 1 \\
Mean s.d.u & $\frac{1}{600}$ & $\frac{1}{600}$ \\
transmission time $\left(\tau\left[\frac{s}{s . d . u}\right]\right)$ & 200 & 150 \\
Number of IoT-SP Sinks $\left(N_{1}\right)$ & 100 & 150 \\
Number of IoT-SP 2 Sinks $\left(N_{2}\right)$ & 100 \\
\hline
\end{tabular}

Table 2 Game I solutions

\begin{tabular}{lcc}
\hline Game I Variable & Scenario 1 & Scenario 2 \\
\hline OP price $\left(p\left[\frac{m . u}{s . d .}\right]\right)$ & 0.5 & 0.5 \\
Fraction os sinks subscribed $\left(x_{1}\right)$ & 1 & 1 \\
Amount of IoT-SP ${ }_{1}$ data $\left(R_{1}\left[\frac{s . d . u}{s .}\right]\right)$ & 200 & 150 \\
Amount of IoT-SP $P_{2}$ data $\left(R_{2}\left[\frac{s . d . u}{s}\right]\right)$ & 100 & 150 \\
\hline
\end{tabular}

Table 3 Reference Case - Game II Differential Game parameters

\begin{tabular}{lc}
\hline Parameter & Scenario 1 and 2 Value \\
\hline Number of final users $(M)$ & 1000 \\
Dynamic's learning rate $(\delta)$ & 0.7 \\
Initial social state $\left(X_{u}(0)\right)$ & $\{0.2,0.8\}$ \\
Final time horizon $(T[s])$ & 10 \\
Discount rate $(\eta)$ & 0 \\
\hline
\end{tabular}

method [25], and the results are shown in Section 4. This method requires the discretization of time, nevertheless, this is absolutely transparent for the researchers.

\section{Results and Discussion}

In this section, we present the numerical results for Game II.

The value of Game I parameters for the two scenarios that we analyzed are shown in Table 1, and the solutions of Game I given that parameters are shown in Table 2. On the other hand, Game II is solved using the reference case parameters shown in Table 3 for both scenarios.

\subsection{Scenario 1: IoT-SPs dynamic competition with different number of sinks}

In this subsection we show the results of Game II when each provider has a different amount of sinks deployed, given the solution for Game I shown in Table 2, column "Scenario 1".

Fig. 6 shows the variation of the dynamic prices $\left(f_{1}(t), f_{2}(t)\right)$, compared with the equilibrium prices of the static analysis $\left(f_{1}^{*}\right.$-Static, $f_{2}^{*}$-Static). At the beginning, the providers change their prices, due to the differences between the static equilibrium values and the initial populations. Once the static equilibrium is reached the behavior of the providers is stationary, until $t$ is close to $T$, where the providers increase their prices.

Fig. 7 shows the evolution of the users' social state in the dynamic case $\left(y_{1}(t), y_{2}(t)\right)$, compared with social state 


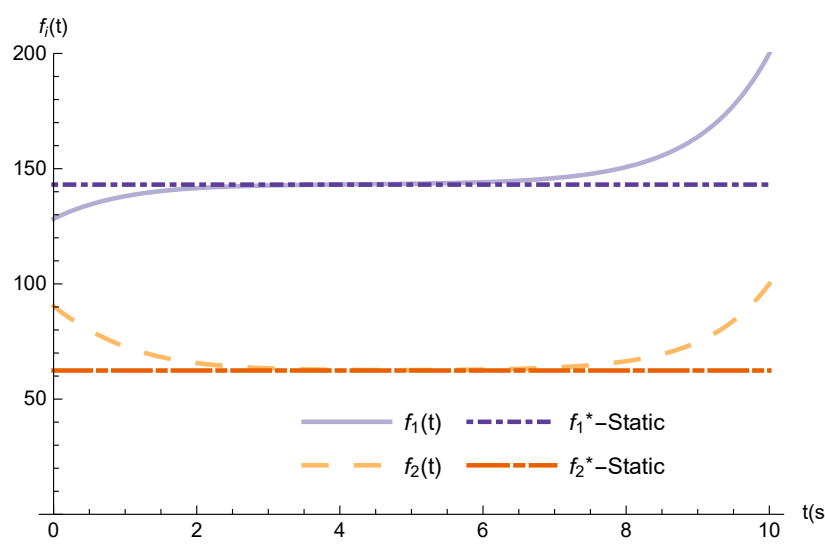

Figure 6 Scenario 1: IoT-SPs equilibrium prices in the dynamic and static approach as a function of $t$.

equilibrium in the static analysis $\left(y_{1}^{*}-\right.$ Static, $y_{2}^{*}-$ Static $)$. At the beginning, the social state evolves due to the difference between the initial and the equilibrium values. Once the social state reaches the equilibrium it remains constant. We do not observe significant changes in the users' social at the end of the time interval, when the prices increase, given that we have not included the option of not subscribing. It is also interesting to note that the provider with more sinks gathering and transmitting data $\left(R_{i}\right)$, is able to fix a higher price and still maintain a higher number of users subscribing to it.

Fig. 8 shows the instantaneous profits in the dynamic case $\left(\Pi_{\mathrm{IoT}_{-\mathrm{SP}_{1}}(t)}, \Pi_{\mathrm{IoT}-\mathrm{SP}_{2}(t)}\right)$, compared with the profits in the static analysis $\left(\Pi_{\mathrm{IoT}_{-} \mathrm{SP}_{1}}-\right.$ Static, $\left.\Pi_{\mathrm{IoT}-\mathrm{SP}_{2}}-\mathrm{Static}\right)$. We observe a similar behavior in the instantaneous profit of the providers, compared with the prices and the social state, where there is a transition stage until the stationarity is reached, and the profit only deviates from the equilibrium when $t$ is close to $T$. The aggregated profit, obtained numerically integrating the instantaneous profit, for the IoT-SP $_{1}$ is 839325 while for the IoT-SP 2 is 298900 . We observe that the profit for the IoT-SP 1 are higher than the profit of the IoT-SP 2 as expected, given that $R_{1}>R_{2}$.

4.2 Scenario 2: IoT-SPs dynamic competition with same number of sinks

In this subsection we analyze the same variables when both providers have the same amount of sinks deployed, given the solution for Game I shown in Table 2, column "Scenario 2".

In this scenario we observe a similar behavior than in Scenario 1, with the difference that given that the two provider have the same amount of sinks, the equilibrium values for the prices, social state and profits are the same for both providers, as shown in Figs. 9, 10 and 11, respectively. In this case, the aggregated profit are 492837 for IoT-SP 1 and 558575 for IoT-SP 2 . They are not the same due to

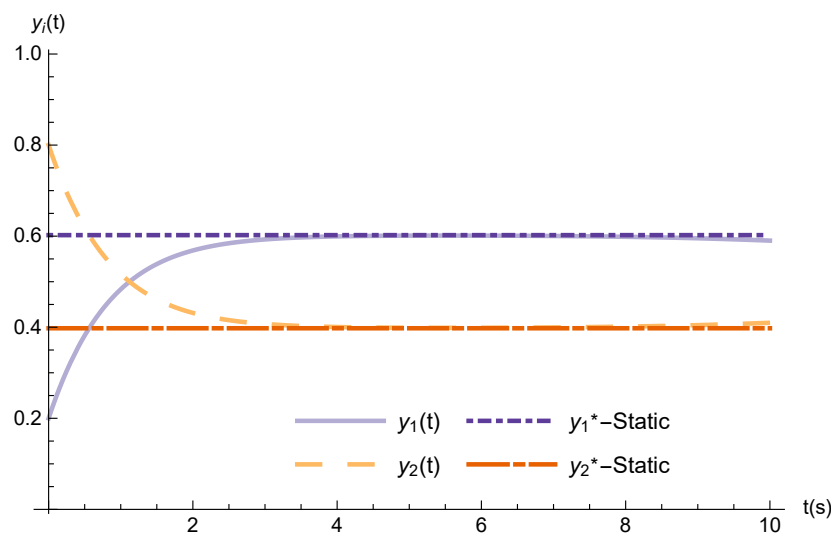

Figure 7 Scenario 1: Social state in the dynamic and static approach as a function of $t$.

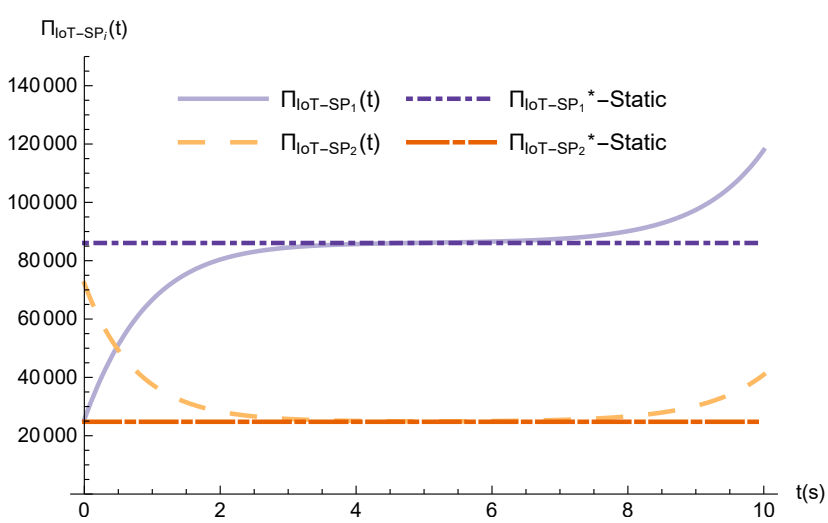

Figure 8 Scenario 1: IoT-SPs profits in the dynamic and static approach as a function of $t$.

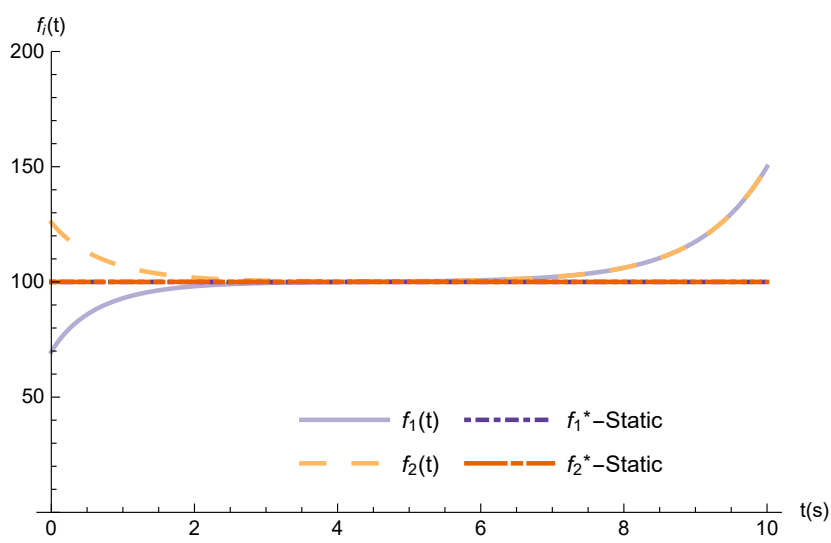

Figure 9 Scenario 2: IoT-SPs equilibrium prices in the dynamic and static approach as a function of $t$.

the differences between the initial state of the population of users and the equilibrium. It is interesting to point out that the dynamic approach is able to evolve from any initial population and reach the stationarity, that matches with the Nash equilibrium of the static approach. In addition, the dynamic approach is also able to choose the best strategies in scenarios where the parameters of the system, such as the number of sinks deployed or the number of users, evolve. 


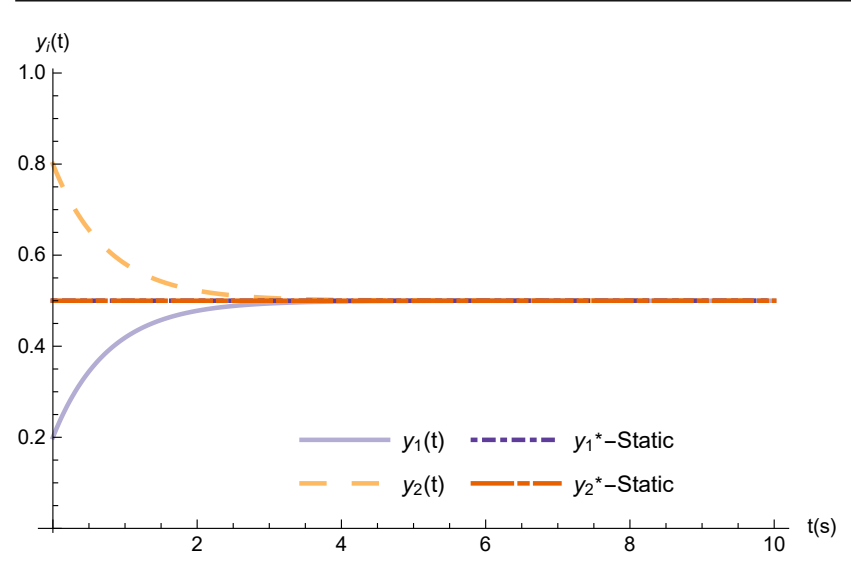

Figure 10 Scenario 2: Social state in the dynamic and static approach as a function of $t$.

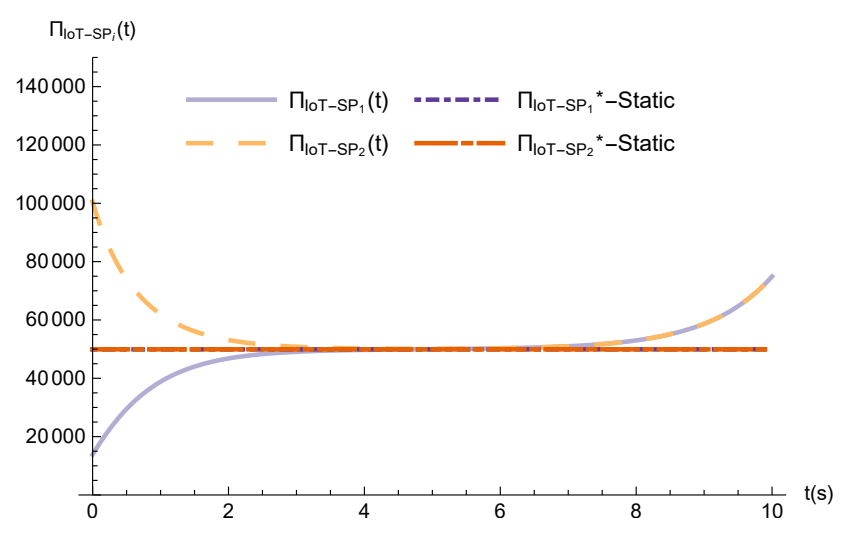

Figure 11 Scenario 2: IoT-SPs profits in the dynamic and static approach as a function of $t$.

\section{Conclusions}

The dynamic provision of services based on wireless sensor-data, has been studied. The model was studied using game theory, evolutionary games, Logit dynamic, maximization and differential games.

Firstly, the model was analyzed as two games. The first game analyzed the interaction between the OP and the sinks, while the second game analyzed the competition between IoT-SPs to provide services to final users and the behavior of the users. The first game was considered static from the point of view of the users and IoT-SPs, given that the frequency of the changes in that game is much lower than in the second game.

Secondly, a Logit dynamic was chosen to model users' behavior, while the competition between IoT-SPs in order to maximize their profit was analyzed using a differential game.

It has been shown that the competition in prices is economically viable for both operators, as shown in the stationary values of the profits and that the dynamic pricing is possible and converges to the static equilibrium. In addition, we have observed that the competition between providers keeps the prices bounded until time is close to the end of the interval, therefore, it is recommended to introduce a reservation value or an infinite interval in order to observe the long term behavior. We also have shown how the provider with more sinks is able to fix a higher price, to keep a higher portion of subscribers and therefore, to obtain a higher profit. Finally, it has been shown how the initial distribution of users alters the profit of two competing providers with the same number of sensors in short term optimizations.

\section{References}

1. L. Tan, Future internet: The Internet of Things, 3rd Int. Conf. Adv. Comput. Theory Eng. 5, V5 (2010). DOI 10.1109/ICACTE.2010.5579543

2. Ericsson. Ericsson Mobility Report: 70 percent of world's population using smartphones by 2020 (2015)

3. Ericsson. Ericsson Mobility Report (2017). DOI 10.3103/S0005105510050031

4. A. Biral, M. Centenaro, A. Zanella, L. Vangelista, M. Zorzi, The challenges of M2M massive access in wireless cellular networks, Digit. Commun. Networks 1(1), 1 (2015). DOI 10.1016/j.dcan.2015.02.001

5. M.R. Palattella, M. Dohler, A. Grieco, G. Rizzo, J. Torsner, T. Engel, L. Ladid, Internet of Things in the 5G Era: Enablers, Architecture, and Business Models, IEEE J. Sel. Areas Commun. 34(3), 510 (2016). DOI 10.1109/JSAC.2016.2525418

6. Z. Mao, G. Nan, M. Li, A Dynamic Pricing Scheme for Congestion Game in Wireless Machine-to-Machine Networks, Int. J. Distrib. Sens. Networks 2012, e840391 (2012). DOI 10.1155/2012/840391

7. E.E. Tsiropoulou, P. Vamvakas, S. Papavassiliou, Joint Customized Price and Power Control for Energy-Efficient Multi-Service Wireless Networks via S-Modular Theory, IEEE Trans. Green Commun. Netw. 1(1), 17 (2017). DOI 10.1109/TGCN.2017.2678207

8. M. Koseoglu, in IEEE 2nd World Forum on Internet of Things (WF-IoT) (2015), pp. 187-192. DOI 10.1109/WF-IoT.2015.7389050

9. J.R. Vidal, V. Pla, L. Guijarro, J. Martinez-Bauset, Dynamic spectrum sharing in cognitive radio networks using truthful mechanisms and virtual currency, Ad Hoc Networks 11(6), 1858 (2013). DOI 10.1016/j.adhoc.2013.04.010

10. Phillips, Teaching Pricing and Revenue Optimization, INFORMS Trans. Educ. 4(1), 1 (2003). DOI 10.1287/ited.4.1.1

11. M. Mandjes, Pricing strategies under heterogeneous service requirements, Comput. Networks 42(2), 231 (2003). DOI 10.1016/S1389-1286(03)00191-9 
12. S. Jara-Díaz, Transport Economic Theory (Emerald Group Publishing Limited, 2007)

13. J. Romero, L. Guijarro, V. Pla, J.R. Vidal, Price competition between a macrocell and a small-cell service provider with limited resources and optimal bandwidth user subscription: a game-theoretical model, Telecommun. Syst. (2017). DOI 10.1007/s11235-017-0331-2

14. D. Niyato, E. Hossain, Competitive pricing for spectrum sharing in cognitive radio networks: Dynamic game, inefficiency of nash equilibrium, and collusion, IEEE Trans. Wirel. Commun. 26(1), 192 (2008). DOI 10.1109/JSAC.2008.080117

15. D. Niyato, E. Hossain, Competitive Pricing in Heterogeneous Wireless Access Networks: Issues and Approaches, IEEE Netw. 22(6), 4 (2008). DOI 10.1109/MNET.2008.4694168

16. A. Al Daoud, M. Alanyali, D. Starobinski, Pricing strategies for spectrum lease in secondary markets, IEEE/ACM Trans. Netw. 18(2), 462 (2010). DOI 10.1109/TNET.2009.2031176

17. K. Zhu, D. Niyato, P. Wang, Z. Han, Dynamic spectrum leasing and service selection in spectrum secondary market of cognitive radio networks, IEEE Trans. Wirel. Commun. 11(3), 1136 (2012). DOI 10.1109/TWC.2012.010312.110732

18. K. Zhu, E. Hossain, D. Niyato, Pricing, spectrum sharing, and service selection in two-tier small cell networks: A hierarchical dynamic game approach, IEEE Trans. Mob. Comput. 13(8), 1843 (2014). DOI 10.1109/TMC.2013.96

19. P.K. Chintagunta, V.R. Rao, Pricing Strategies in a Dynamic Duopoly: A Differential Game Approach, Manage. Sci. 42, 1501 (1996)

20. A. Sanchis-Cano, J. Romero, E. Sacoto-Cabrera, L. Guijarro, Economic Feasibility of Wireless Sensor Network-Based Service Provision in a Duopoly Setting with a Monopolist Operator, Sensors 17(12), 2727 (2017). DOI 10.3390/S17122727

21. E. Altman, T. Boulogne, R. El-Azouzi, T. Jiménez, L. Wynter, A survey on networking games in telecommunications, Computers and Operations Research 33(2), 286 (2006). DOI 10.1016/j.cor.2004.06.005

22. P. Reichl, S. Egger, R. Schatz, A. D'Alconzo, in IEEE Int. Conf. Commun. (IEEE, 2010), pp. 1-5. DOI 10.1109/ICC.2010.5501894

23. T. Weber, Optimal Control Theory with Applications in Economics (The MIT Press, 2011). DOI 10.7551/mitpress/9780262015738.001.0001

24. W.H. Sandholm, Population games and evolutionary dynamics (The MIT Press, Cambridge, Mass, 2010)

25. Wolfram. Numerical Solution of Boundary Value 